\title{
DNA Vaccine VB10.16
}

National Cancer Institute

\section{Source}

National Cancer Institute. DNA Vaccine VB10.16. NCI Thesaurus. Code C123923.

A therapeutic DNA vaccine composed of three parts, one encodes the E6/E7 fusion protein of human papillomavirus (HPV) type 16 (HPV16), the second is a dimerization entity and the third part encodes a protein that specifically binds to antigen presenting cells (APCs), with potential immunostimulating and antineoplastic activities. Upon intramuscular administration, the DNA vaccine VB10.16 expresses HPV16 E6/7 and a protein that targets receptors on APCs. Upon binding to APCs and subsequent internalization, the APCs mature and the HPV16 E6/7 antigenic protein is presented by the APCs. This attracts and stimulates B-lymphocytes, CD4-positive T-lymphocytes and elicits a cytotoxic T-lymphocyte (CTL) response against cancer cells expressing HPV16associated E6 and E7 oncoproteins, which result in tumor cell lysis. HPV16 E6/7, a viral antigen, plays a key role in the development of certain types of cancer. 